\title{
A Peer-to-Peer Reference Architecture
}

\author{
Atul Singh and Mads Haahr \\ Distributed Systems Group \\ Department of Computer Science \\ Trinity College, Dublin \\ Ireland \\ Email: Atul.Singh@cs.tcd.ie, Mads.Haahr@cs.tcd.ie
}

\begin{abstract}
Peer-to-Peer (P2P) applications are extremely popular on the Internet because they allow users to share information in a decentralised manner. Internet users use file-sharing applications (e.g., Bit Torrent, KaZaA) to share and exchange files and collaboration applications (e.g., Jabber, Groove) to exchange chat messages and work on shared documents. A reference architecture for a domain provides an architecture template which can be used as a starting point for designing the software architecture of a system in that domain. Despite the popularity of P2P applications there is no reference architecture for the P2P domain. This paper presents a reference architecture for the P2P domain. The reference architecture has been designed to satisfy the concerns that a P2P application needs to address. The reference architecture takes a service-centric view of the P2P domain. The reference architecture can be used to describe the structure of existing P2P applications and middlewares. The paper validates the reference architecture by describing the structure of some of the existing P2P applications and middlewares using the reference architecture.
\end{abstract}

\section{INTRODUCTION}

P2P applications are distributed systems without any central control, where all the peers are equivalent in functionality. Recent surveys show that P2P applications generate a high percentage of traffic on the Internet [1]. The high popularity of P2P applications among Internet users is because of its ability to allow its users to share and exchange information without the need of costly and difficult to maintain centralized infrastructure. Internet users use file-sharing applications (e.g., BitTorrent [2], $\mathrm{KaZaA}$ [3]) to share and exchange files and collaboration applications (e.g. Jabber [4], Groove [5]) to exchange chat messages and work on shared documents. Despite the popularity of $\mathrm{P} 2 \mathrm{P}$ applications there is no reference architecture for the $\mathrm{P} 2 \mathrm{P}$ domain. This paper presents a reference architecture for the $\mathrm{P} 2 \mathrm{P}$ domain.

A software architecture describes the structure of a software system. It describes and defines the software elements that comprise the system, the externally visible properties of these elements, and the relationships among them [6]. Software architectures are used to communicate the high-level design of a system to a diverse audience which may include programmers, managers and customers. The software architecture design phase is used to take design decisions which affect a system's remaining development, deployment and maintenance life.

A reference architecture defines the software elements, their functional responsibilities and the allowed interactions between them for a particular domain [7], [6], [8]. Reference architecture can be used as the starting point for designing the architecture of a software system of that domain. A reference architecture saves time and effort in the areas of requirement analysis and functional partitioning of the system [9]. Reference architecture is often used in mature domains like compilers and databases. For example a compiler is composed of a scanner parser, semantic analyzer and a code generator subsystem [10]. The dramatic success of Napster [11] in 1999, brought P2P applications into the limelight. Since then the research community has done a lot of work on P2P and many new P2P applications have been developed. Wikipedia [12] lists more than one hundred P2P applications. The authors feel that the P2P domain has reached a level of maturity that a reference architecture should be established for it. This paper proposes a possible reference architecture for the $\mathrm{P} 2 \mathrm{P}$ domain.

The reference architecture presented in this paper has been derived by studying existing $\mathrm{P} 2 \mathrm{P}$ middlewares and applications. Based on this we have identified the core-concerns (use cases) that a P2P application needs to address. The elements of the reference architecture cooperate to satisfy these core concerns. A service-centric view has been taken to simplify the reference architecture because the notion of services lends itself well to modelling P2P applications and middlewares. A P2P overlay network is used to share both resources and services among peers on the overlay network. However resources can be accessed through services and so the reference architecture presented in this paper takes a servicecentric view. In the reference architecture peers on the overlay network work together to use services offered by each other. The shared services can be used to access resources shared on the overlay network. A service-centric view facilitates the development of P2P applications and middlewares using the reference architecture because the software implementation can use the support for service-oriented architecture provided by the existing middlewares like CORBA [13] and SOAP [14].

Bernstein [15] defines middleware as programming interfaces and protocols that sit "in the middle", in a layer above the operating system and networking software and below industry specific applications. A middleware provides services that can be used to rapidly develop and deploy distributed applications. Singhai [16] has done research on describing the structure of existing object-oriented middlewares like CORBA and Java RMI [17]. The growing popularity of P2P applications 


\begin{tabular}{l||l}
\hline $\begin{array}{l}\text { Component-Connector } \\
\text { element }\end{array}$ & $\begin{array}{l}\text { UML 2.0 } \\
\text { element }\end{array}$ \\
\hline Component & Component \\
\hline Connector & Associations \\
\hline Role & Not represented \\
\hline Port & Port \\
\hline Protocol & Note \\
\hline
\end{tabular}

TABLE I

THE UML 2.0 ELEMENTS USED TO DRAW COMPONENT-CONNECTOR DIAGRAMS

has given rise to P2P middlewares (e.g. JXTA [18], MSN P2P [19]). While these middlewares facilitate P2P application development, there is a lack of a common vocabulary to effectively describe the structure of these middlewares and $\mathrm{P} 2 \mathrm{P}$ applications. The reference architecture presented in this paper can be used to describe and compare the structure of P2P applications and middlewares. This paper uses the reference architecture to describe the structure of some popular P2P applications and middlewares. The reference architecture can be used as a starting point for developing the software architecture of new P2P applications and middlewares. The reference architecture can be used to develop a customizable P2P middleware which allows instances of different types of architecture elements in the reference architecture to work together with each other to create a P2P application.

The rest of the paper is organized as follows. Section II presents the background material that is required to understand the work presented in this paper. The section defines Service Oriented Architecture (SOA) and discusses the rationale behind taking a service centric view. The section also presents the diagrammatic notation used to describe the reference architecture. Section III presents a summary of the study of existing $\mathrm{P} 2 \mathrm{P}$ applications and middlewares which is used as the basis for deriving the reference architecture. Section IV presents the reference architecture. Section V validates the reference architecture by describing the structure of some of the existing $\mathrm{P} 2 \mathrm{P}$ applications and middlewares using the reference architecture. Section VI presents the conclusion of this work.

\section{BACKGROUND}

\section{A. Service-Oriented Architecture}

A service is a unit of work done by a service provider to achieve desired results for a service consumer [20]. Serviceoriented architectures (SOA) is an approach that utilizes software services as fundamental elements for developing software applications [21], [20], [22]. In SOA software resources are services available and discoverable on a network. Common Object Request Broker Architecture (CORBA), Microsoft's Distributed Component Object Model (DCOM) and SOAP are examples of middlewares that provided this functionality.

In SOA the underlying implementation details of a service are hidden from the service consumer through encapsulation. There is a loose coupling between the service provider and the service consumer which means that changes can be done in either one of them without affecting the other. The service consumer uses the service interface description and the service address to access the service. The service provider can easily change the implementation of the service without breaking the service consumer. Also, if required, the service consumer can use a service at an alternate location if it has same service interface as the original service provider. The loose coupling between the service provider and consumer facilitates the use of the same service across many applications, thereby reducing the development cost and errors in the application and ensuring faster development time.

The reference architecture uses the service-oriented architecture because it lends itself well to modelling P2P applications and middlewares. A P2P system is used to share a wide variety of resources and services. A P2P system can be used to access shared services which perform tasks like providing information about other peers on the network, numerical computations and routing of messages on the overlay network. A P2P system can be used to access a wide variety of shared resources like files, videos, messages, CPU cycles and storage space. However the shared resources can be accessed through services running on the peers. Since all the sharing in a P2P system can be conceptualized using services, a serviceoriented approach has been used in the reference architecture. The reference architecture is designed to support sharing and access of services on a P2P network.

The paper uses the term servent ${ }^{1}$ to refer to components which provide a service and can be used to access a similar service provided by another peer. A servent can act both as a client and a server. All the peers in a P2P network are equivalent in functionality and can act both as a client and a server. A peer needs to act both as a consumer (client) and provider (server) for all the concerns mentioned in Table II because of which the reference architecture handles most of these concerns using servents.

Peers join a P2P network to utilize services offered by other peers. The services can be divided into two types: network service (NES) and node service (NOS). A network service is available on more than one peer in the network. Different instances of the network service work together to execute a task. A node service is offered by an individual peer. The service is specific to the peer offering the service. This distinction is important as it determine the way a service will be invoked.

\section{B. Documenting Software Architecture}

Software architecture is documented using views, each of which concentrates on a different aspect of the software system. Different researchers suggest using different types and numbers of views for documenting software architectures. For example [23] suggest using 4+1 views, [6] suggest using 3 views and Soni et. al. in their works [7], [24], [25] suggest using 4 views. This work follows the suggestion given by Soni et.

\footnotetext{
${ }^{1}$ coined by merging together the first three letters of the word server and the last three letters of the word client
} 
al.. They suggest using four views called code view, execution view, module view and conceptual view to document a software architecture. The views are enumerated in the reverse order in which they should be designed. The first three views deal with the actual implementation of the software system using programming languages, operating systems, communication mechanisms and so forth.

The fourth view or the conceptual view describes the structure of the software system at a high level of abstraction using architecture elements which can not be directly implemented by using software technology [7]. In this view the functionality of the system is mapped to architecture elements called conceptual components (or just components), with coordination and data exchange handled by elements called connectors. Both components and connectors can be further decomposed into more components and connectors. The notion of building a system by interconnecting components is appealing because of the potential for reuse and for incorporating off-theshelf components. The components interact with the outside world using elements called ports. A port describes both the messages (operations) that the component can process and the messages that it invokes. Connectors interact with the components using elements called roles. Both ports and roles obey protocols. A protocol is defined as a set of incoming message types, outgoing message types and the valid message exchange sequence. A port and a role can be connected together if the port's protocol is the conjugate of the role's protocol. Because of space constraints this paper only presents the conceptual view of the reference architecture.

The diagram used to describe a conceptual view is called a component-connector (CC) diagram. A CC diagram shows the elements (e.g. components, connectors ) of the conceptual view. The paper uses the UML 2.0 standards to draw the CC diagrams. There is no direct mapping between the elements of the component-connector diagram and the UML notation [26]. Table I shows the UML elements that have been used to draw the elements of CC diagrams. UML components can contain other UML elements which makes them an ideal choice for representing $\mathrm{CC}$ components. The newly introduced concept of ports in UML 2.0 is similar to the ports in CC diagrams and has been used to represent the ports. The paper uses UML associations to represent connectors as suggested in [26]. Since roles and ports which are connected to each other must obey similar protocols, roles are not documented in the $\mathrm{CC}$ diagrams in this paper.

\section{CORE-CONCERNS FOR A P2P APPLICATION}

Current P2P applications can be divided into three major categories based on application domain: parallel computing, content-management and collaborative [27]. The authors have studied P2P middlewares and applications belonging to all the three major categories of $\mathrm{P} 2 \mathrm{P}$ applications and have identified the concerns that a P2P application needs to address [28]. The concerns can be divided into five groups. Table II presents the groups along with the concerns. The concerns are the usecases that a P2P application needs to address. The reference

\begin{tabular}{l||l}
\hline \multicolumn{1}{l}{ Group } & Concern \\
\hline \multirow{2}{*}{$\begin{array}{l}\text { Naming } \\
\text { Manerlay }\end{array}$} & Identification of entities in a P2P system (identification). \\
\cline { 2 - 2 } & $\begin{array}{l}\text { Resolution of entity id to a physical address (resolution). } \\
\text { Search for a connected peer on the overlay network } \\
\text { (search). }\end{array}$ \\
\cline { 2 - 2 } & Handle join requests (join). \\
\hline \multirow{2}{*}{$\begin{array}{l}\text { Service } \\
\text { Management }\end{array}$} & Handle leave requests (leave). \\
\cline { 2 - 2 } $\begin{array}{l}\text { Message } \\
\text { Routing }\end{array}$ & Advertise services/resources to share (advertise). \\
\cline { 2 - 2 } Security & Access the service/resources (Access). \\
\cline { 2 - 2 } & Maintain the topology (topology). \\
\cline { 2 - 2 } & Routing of messages (routing). \\
\cline { 2 - 2 } & $\begin{array}{l}\text { Authentication of peers (authentication). } \\
\text { (authorization of peers to access a resource/service }\end{array}$ \\
\cline { 2 - 2 } & Secure transmission of messages (confidentiality). \\
\hline
\end{tabular}

TABLE II

THE CORE CONCERNS THAT A P2P APPLICATION NEEDS TO ADDRESS.

architecture presented in this paper handles all these use-cases.

P2P systems assign a network (underlying physical network) and location independent id (identification) to the entities (e.g., peers, resources, services, etc.) in a system. The id's allow $\mathrm{P} 2 \mathrm{P}$ systems to manage entities whose physical location (IP address) on the network changes with time. The id's are dynamically resolved (resolution) to determine the current physical location of the entity. For example, in the JXTA [18] reference implementation the id is a 128 bit universally unique identifier (UUID) generated by the peer. The JXTA reference implementation uses a combination of a central server (called rendezvous server) and IP multicast to resolve the id to a physical address.

Peers join an overlay network through another peer which is already connected to the overlay network. Connected peers' addresses may be well known information or they can be obtained by IP multicast (search). The peer accepting the connection (join) may redirect the incoming peer to another peer. The connecting peer may also be supplied with information relevant for it. For example, in Jabber [29], [4], [30] peers connect to a well known server to join the overlay network. The server provides the connecting peer with information about the availability of other peers on the network.

Peers use the overlay network to advertise their resources and services and to discover new resources and services which they can access. For example, in Jabber the peers advertise the details of the shared resources and services to a central server. Peers can discover the details about the shared services/resources by sending a query message to the central server. The service/resources can be accessed by doing a remote process call (RPC). Peers may inform one or more peers on the overlay network when they leave the overlay network. For example, in Jabber the peers send a presence message of type unavailable to the central server.

Peers in decentralized P2P systems maintain connections to other peers in the network. The graph formed by these connections constitutes the topology of the overlay network. The connections are used to route messages. A peer can directly send the message to the destination peer if the 


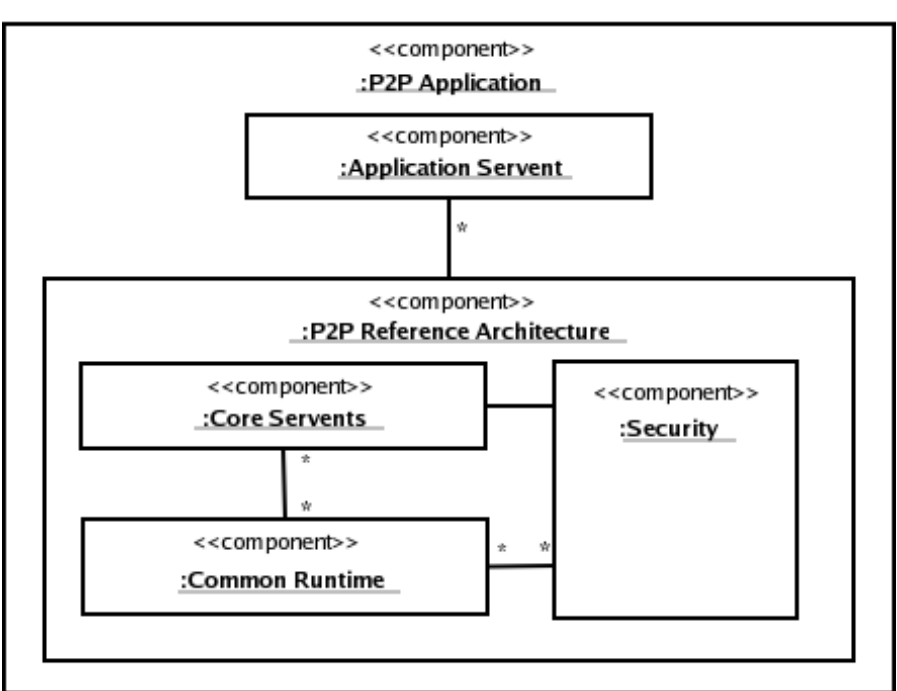

Fig. 1. A UML diagram providing a high level overview of the P2P reference architecture.

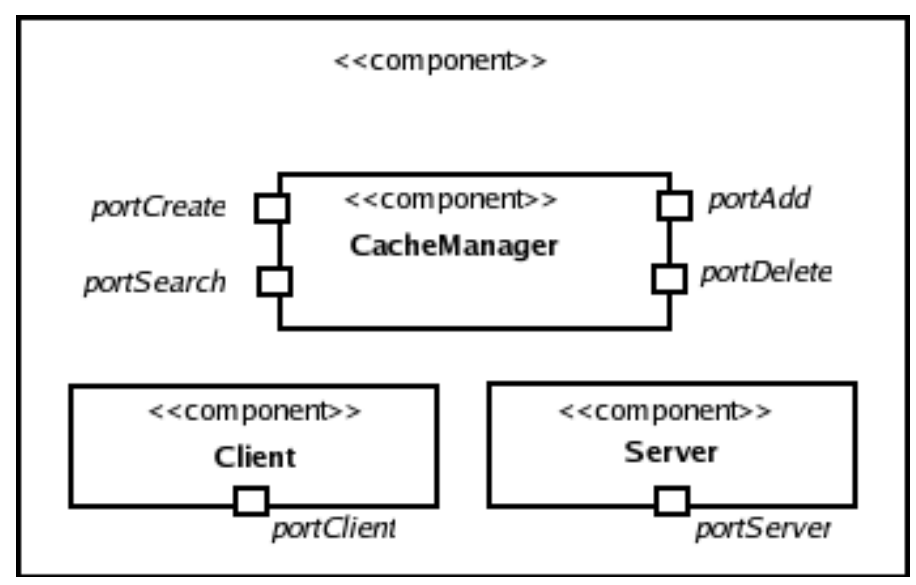

Fig. 2. A UML diagram presenting a conceptual view of the runtime component.

destination peer's address is available. If the destination peer is not accessible or its physical address is not available then the source peer may route the message to one or more known peers on the network, which can in turn route the message to the destination peer. The P2P software is responsible for maintaining connections (topology) which improve the efficiency of the P2P system. Authentication, authorization and secure transmission of messages (confidentiality) are the security-related concerns.

\section{P2P REFERENCE ARCHITECTURE}

Figure 1 presents a high-level overview of the reference architecture. The reference architecture consists of three key components: common runtime, security and core servents. The common runtime provides the messaging infrastructure used to exchange messages between peers in order to invoke

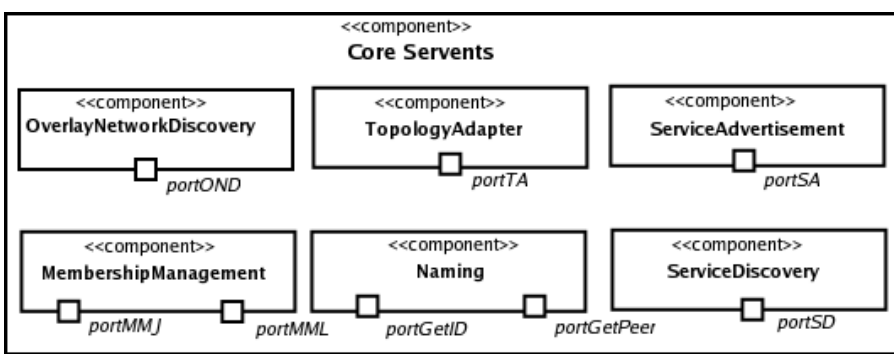

Fig. 3. A UML diagram depicting the conceptual view of the core servents of the reference architecture. The core servents handle most of the concerns which need to be addressed by a P2P application.

(access) a service. The common runtime is used by all types of servents to communicate with servents on other peers. The security component deals with handling the security related concerns such as authentication, authorization and the secure transmission of messages. The reference architecture suggests using aspects [31], [32] to implement the security-related concerns. This paper does not define the structure of the security components. Except for routing, the core servents address all the concerns that a $\mathrm{P} 2 \mathrm{P}$ application needs to handle. The routing logic may vary for different services and is handled by the servent for which a request arrives. In the reference architecture a P2P application consists of application servents (e.g., for file sharing or instant messaging) that are responsible for the application logic. The reference architecture supports multiple co-existing application servents. This means that an application developed using the reference architecture can be used to share multiple services. An instance of the reference architecture can have multiple instances of the core servents, security component and runtime. These instances can be used by an application to connect to different overlay networks.

There are six core servents (see Table IV) which handle all the concerns except routing and access. Figure 3 shows a conceptual view of the core servents. The core servents along with the concerns they handle are shown in Table IV. A servent can handle incoming messages from both remote and local servents and applications. The protocol used by the core servent ports is shown in Table III. The protocols are documented using the notation suggested in [7]. The table does not show implementation-specific details (e.g. source IP and port of message that are sent with the messages). The protocols define the structure of the messages exchanged to invoke these servents. The OverlayNetworkDiscovery (OND) servent is used to search for connected peers on the overlay network to which a new peer can connect. The port portOND of the OND servent expects a single incoming message getPeers with the new peer's information and responds with information about a list of peers to which the new peer can connect. The MembershipManagement (MM) servent is used to handle requests to join and leave the overlay network. The port portMMJ of the MM servent expects an incoming message joinOverlayNetwork with the new peer's information. Upon receiving the joinOverlayNetwork message the servent 


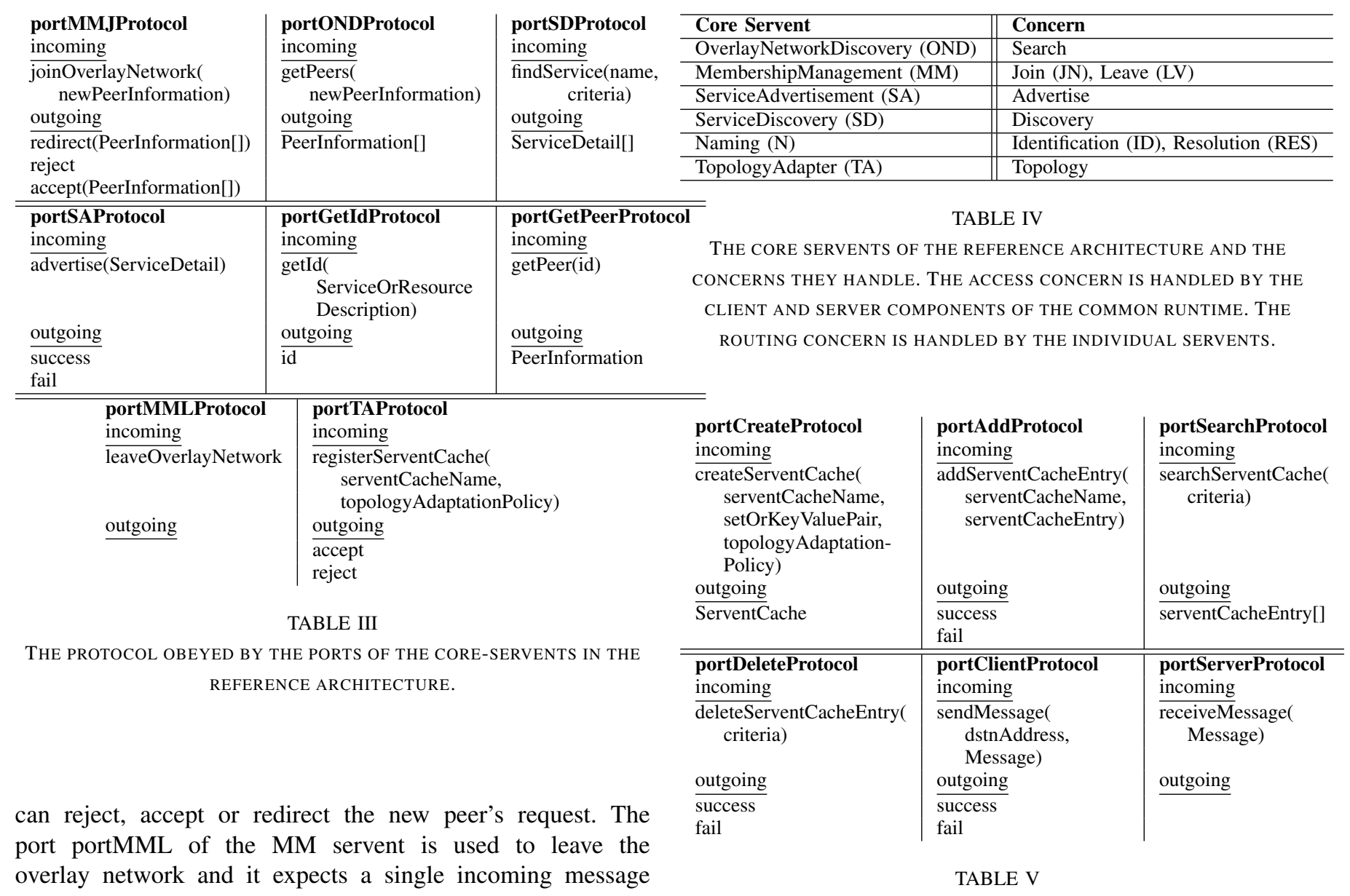

called leaveOverlayNetwork. A peer wishing to advertise its services sends an advertise message to the port portSA of its ServiceAdvertisement (SA) servent. The SA servent may advertise the service detail to the SA servent of the other peers on the overlay network and responds back to its peer with a success or fail message. The ServiceDiscovery (SD) servent is used to discover the details of services on the overlay network that match a search criterion. The portSD port of the SD servent expects a single incoming message findService with the search criteria and responds with the details of a list of services matching the search criteria. The Naming (N) servent is used to assign a unique identifier to entities in a P2P system (using portGetID) and to resolve an entity's id to a physical IP address (using portGetPeer).

An overlay network can be defined in terms of the policies it uses to implement the different components of the reference architecture. A peer can join an overlay network if it has the set of instances of the reference architecture elements that implement the policies that the overlay network desires. Existing P2P systems like JXTA [33] try to make P2P applications interoperable by defining standards (format and on wire form of the message) for the messages exchanged between peers. However messaging standards alone do not ensure interoperability. For example, a peer (P) might not implement the SA servent and instead use a multicast on the overlay network policy for the SD servent to discover services on the overlay network. An overlay network $(\mathrm{ON})$ with a small

number of peers might expect its peers to use multicast to all known peers policy for the SA servent and to use the information provided by the SA servent to perform service discovery (SD). When $\mathrm{P}$ joins $\mathrm{ON}$, messaging standards for example those set by JXTA, ensure that peers on ON can understand the SD messages send by P. However the peers on ON will not know how to process the messages sent by peer $P$.

The common runtime can be further decomposed into three components (see Figure 2) which are: Client, Server and CacheManager. The server component receives messages from the network and directs them to the appropriate servent. The client component helps servents to invoke services provided by servents on other peers. Together the Client and the Server components handle the access concern. The CacheManager component is responsible for maintaining a cache called the servent cache. The servent cache can be used to process both incoming and outgoing requests for a service and/or provide identifiers of a set of hosts which provide that service. The servent cache can be implemented as a set of values or as a table of (key, value) pairs. The table may be hashed to improve efficiency. A servent instance may ask the CacheManager to create a servent cache of a desired type (set or table). The 


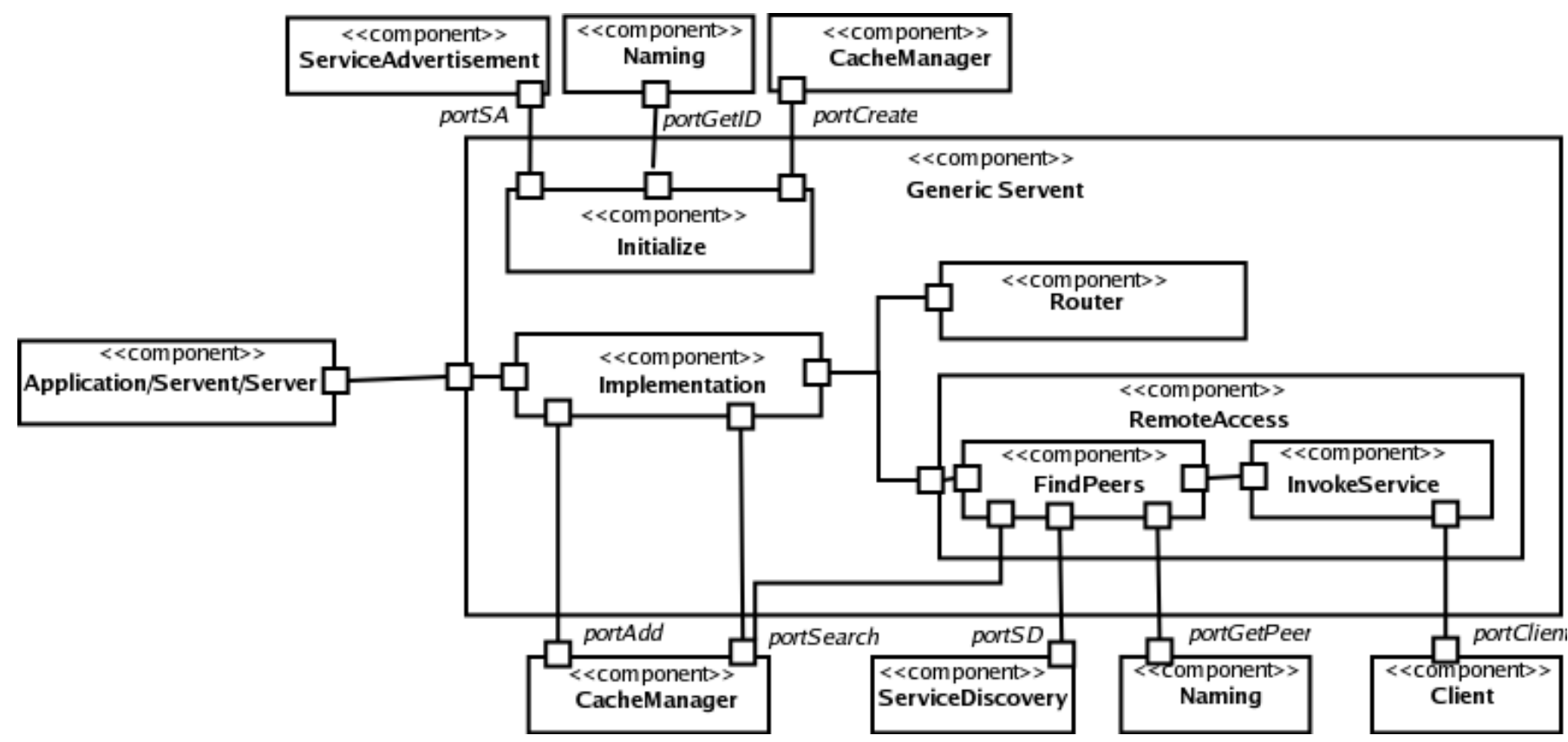

Fig. 4. A UML diagram of the conceptual view of a generic servent. All the connectors depict local method invocation. The Routing component may use the Client component.

data stored in a servent cache will vary with the service. For example, for a file sharing service the servent cache can be a set of (file id, host) pairs. One servent cache can be shared across multiple servents.

Table $\mathrm{V}$ shows the protocols used by the ports of the common runtime components. The port portCreate of the CacheManager component is used to create a servent cache. In the incoming message, the port expects a name for the servent-cache, the topology adaptation step to be used on the servent cache and the description of the servent cache (set or table of (key, value) pairs). The port creates a new servent cache if one with the given name and properties does not exists and returns a reference to it. The portAdd, portDelete and portSearch ports of the CacheManager are used to add, remove and search entries from a servent cache with a given name. The portClient port of the Client component is used to send a given Message to a destination address. The Message format is determined by the protocols obeyed by the port to which the message is destined. The portServer of the Server component is used to receive messages. The server directs an incoming messages to an instance of the servent type (specified in destination Address) for which the message is intended.

The TopologyAdapter servent works on the servent cache. It will normally be used to change the servent cache entries so that a service request can be processed more efficiently. A servent cache would generally contain information about other peers on the overlay network which provide a similar service. A request for a service would be redirected to one or more of these peers if the local servent handling the request can not handle it. The TopologyAdapter servent ensures that the servent cache contains entries which will help in an optimal processing of the service.

Figure 4 shows the conceptual view of a generic P2P servent. During initialization, a servent may do either of the following: i) advertise its services using the port portSA of the ServiceAdvertisement servent and/or ii) use the port portGetId of the naming servent to get an id for the servent, and iii) use the port portAdd of the CacheManager to create a servent cache. The Implementation component provides the actual service implementation. The Implementation component uses the CacheManager to find an existing response for an incoming request from a servent cache (if used by the servent). If a response is available from the CacheManager then it is returned. However if a cached response is not available then the Implementation component tries to process the request locally and send a response. If it can not process the request locally then it uses the RemoteAccess component that uses other peers on the overlay network to process the request and generate a response. The Implementation component directs a request to the Router component for processing if the request's destination is not the servent's peer. Before returning the response the Implementation component updates the serventcache (if used by the servent) using the CacheManager.

The RemoteAccess component of a servent is used to process a service request by using other peers on the overlay network if it can not be processed locally by the servent. The RemoteAccess component uses its FindPeers component to find details of peers hosting similar servents that can be used to process the request. The FindPeers component uses the CacheManager and/or the ServiceDiscovery servent to find these peers. The FindPeers component uses the port portGetPeer of the Naming servent to resolve a peer id to 
its IP address. The InvokeService component then processes the incoming request using the servents on the peers supplied by the FindPeer component.

\section{Applying the Reference Architecture.}

All the components of the reference architecture are abstractions that can have multiple instances implemented using different policies. This section describes some of the existing $\mathrm{P} 2 \mathrm{P}$ applications and middlewares using the reference architecture. This section describes: Gnutella a pure P2P filesharing system, Freenet a P2P content storage system, JXTA a multi-purpose P2P middleware and Jabber a platform for P2P collaboration. For each P2P system the routing algorithm and the servent cache structure is described. The routing policy and the servent cache used by all the servents in the P2P system is documented using tables.

\section{A. Gnutella}

Gnutella [34], [35] is a decentralized P2P file-sharing network. Gnutella creates an unstructured, self-organizing overlay network of peers. There is no constraint on the position of the files. A peer uses a breadth-first search on the overlay network to locate a file. Once the peer containing the file is located, a direct connection is used to transfer the file.

All the peers in Gnutella implement two application servent which are: File getFile(String fileName) which is used by other peers to download files and PeerInformation getPeers() which is a network service used by a connected peer to discover other peers on the overlay network. Table VI shows the policies used by Gnutella to implement the different core-servents. The table also describes the routing policy and the servent cache used by the different servents. New peers join the Gnutella network using an available peer from a list of well-known peers which are connected to the Gnutella overlay network. When the new peer joins the network the peer accepting the connection provides the new peer with information about other peers on the network. Naming service is provided by the Gnutella application. The application generates a unique 16 byte identifier for each new messages.

ServiceDiscovery is implemented as a network service. The ServiceDiscovery, OverlayNetworkDiscovery and getPeers() servents share a single servent-cache which is a set of addresses of the peers on the overlay network. A peer searches for a file it wants by using the ServiceDiscovery servent. The ServiceDiscovery servent sends out a multicast message containing a search request for a getFile service which can provide a file matching the peer's requirements to all the peers in the servent-cache. A peer receiving the request responds back with its address and a list of files matching the search query. If the time to live for the request has not elapsed then the peer forwards the request to all the nodes in its serventcache. The getPeer() network service is accessed by sending a multicast to all the peers in the servent-cache. The peer receiving the request responds back with information about itself. If the request has not reached its time to live then the peer propagates the request to the peers in its servent-cache.

\begin{tabular}{|c|c|c|c|c|}
\hline \multicolumn{5}{|c|}{$\begin{array}{ll}\text { Servent Cache } \\
\end{array}$} \\
\hline \multicolumn{2}{|l|}{ Name } & \multicolumn{3}{|c|}{ Description } \\
\hline \multicolumn{2}{|c|}{ PeerCache (GPC) } & \multicolumn{3}{|c|}{ Set of Peer Information } \\
\hline \multicolumn{5}{|c|}{ Routing Policy } \\
\hline \multicolumn{5}{|c|}{ Name: Multicast (GMC) } \\
\hline \multicolumn{5}{|c|}{$\begin{array}{l}\text { Algorithm: } \\
\text { if (isRequest(message)) }\{ \\
\text { if (!hasSeen(message.id)) }\{ \\
\text { message.TTL (Time to live) = message.TTL - } \\
\text { message.TTL ! = } 0 \text { ? forwardMessage(GPC - } \\
\text { sendMessageResponse(message.peer) } \\
\text { cache(message.id and message.peer mapping) } \\
\} \text { else }\{ \\
\text { if (hasSeen(message.id)) }\{ \\
\text { forwardMessage(findPeerWhichSendRequest(n } \\
\}\end{array}$} \\
\hline \multicolumn{5}{|c|}{ Application Servents } \\
\hline \multicolumn{3}{|l|}{ Name } & $\begin{array}{l}\text { Routing } \\
\text { Policy }\end{array}$ & $\begin{array}{l}\text { Servent } \\
\text { Cache }\end{array}$ \\
\hline \multicolumn{3}{|c|}{$\begin{array}{l}\text { PeerInformation getPeers() } \\
\text { File getFile(String fileName) }\end{array}$} & $\begin{array}{l}\text { GMC } \\
\text { none }\end{array}$ & $\begin{array}{l}\text { GPC } \\
\text { none }\end{array}$ \\
\hline \multicolumn{5}{|c|}{ Core Servents } \\
\hline Name & \multicolumn{2}{|l|}{ Policy } & $\begin{array}{l}\text { Routing } \\
\text { Policy }\end{array}$ & $\begin{array}{l}\text { Servent } \\
\text { Cache }\end{array}$ \\
\hline OND & \multicolumn{2}{|c|}{ Well-known peers } & none & GPC \\
\hline MM(Join) & \multicolumn{2}{|c|}{$\begin{array}{l}\text { Peers on the } \\
\text { overlay network }\end{array}$} & none & GPC \\
\hline MM(Leave) & \multicolumn{2}{|l|}{ none } & none & none \\
\hline SA & \multicolumn{2}{|l|}{ none } & none & none \\
\hline SD & \multicolumn{2}{|c|}{$\begin{array}{l}\text { Multicast to peers } \\
\text { in PeerCache }\end{array}$} & GMC & GPC \\
\hline $\begin{array}{l}\text { N(ID) } \\
\text { N(RES) }\end{array}$ & \multicolumn{2}{|l|}{$\begin{array}{l}\text { none } \\
\text { none }\end{array}$} & $\begin{array}{l}\text { none } \\
\text { none }\end{array}$ & $\begin{array}{l}\text { none } \\
\text { none }\end{array}$ \\
\hline TA & \multicolumn{2}{|l|}{ none } & none & none \\
\hline
\end{tabular}

TABLE VI

Describing Gnutella Using the ReFEREnCE Architecture.

\section{B. Freenet}

Freenet [36], [37] is a censorship free P2P content storage and retrieval system. It provides complete anonymity to the contents publisher and user. It reduces the slashdot effect ${ }^{2}$, by caching the data on multiple nodes closer (in terms of node hops) to the contents users. All data stored on Freenet is associated with a key.

All the peers in Freenet implement two application servents which provide network services. They are Data getData(Key k) which returns data for a given key, and void insertData(key, data) which is used to publish data associated with a key. Table VII shows the policies used by Freenet to implement the core servents of the Reference Architecture. The table also describes the routing policy and the servent cache used by the different servents. New peers join the Freenet network using an available peer from a list of well-known peers which are connected to the Freenet overlay network. When a new peer joins the network the existing peers work together to assign

\footnotetext{
${ }^{2}$ Slashdot effect refers to the slowing down or temporarily closing of a web-site because of a heavy influx of web-traffic caused by a mention of the web-site on Slashdot, a popular technology news and information site.
} 


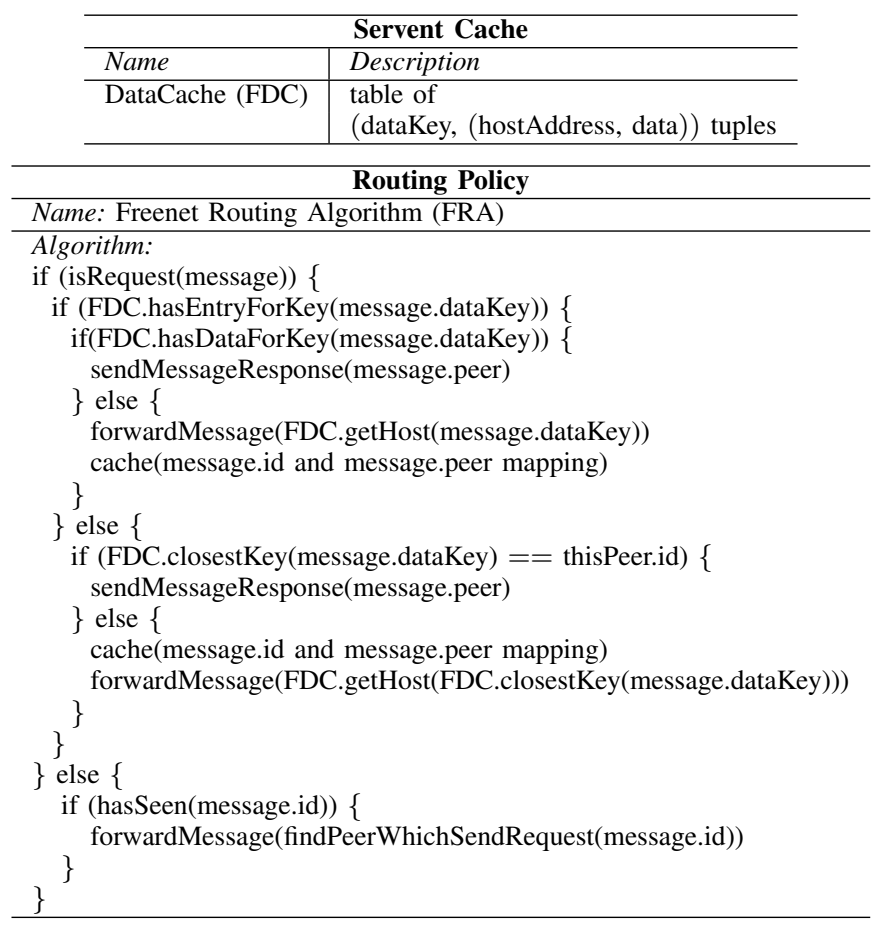

\begin{tabular}{l|l|l|l}
\hline \multicolumn{4}{c}{ Application Servents } \\
\cline { 2 - 4 } Name & $\begin{array}{l}\text { Routing } \\
\text { Policy }\end{array}$ & $\begin{array}{l}\text { Servent } \\
\text { Cache }\end{array}$ \\
\hline $\begin{array}{l}\text { Data getData(Key k) } \\
\text { void insertData(key k, data d) }\end{array}$ & $\begin{array}{l}\text { FRA } \\
\text { FRA }\end{array}$ & $\begin{array}{l}\text { FDC } \\
\text { FDC }\end{array}$ \\
\hline \multicolumn{4}{c}{ Pervents } \\
\hline Name & Policy & $\begin{array}{l}\text { Routing } \\
\text { Policy }\end{array}$ & $\begin{array}{l}\text { Servent } \\
\text { Cache }\end{array}$ \\
\hline OND & Well-known peers & none & none \\
\hline MM(Join) & Key space to manage & FRA & FDC \\
\hline MM(Leave) & none & none & none \\
\hline SA & none & none & none \\
\hline SD & none & none & none \\
\hline N(ID) & $\begin{array}{l}\text { unique id generated } \\
\text { by consulting existing } \\
\text { peers on the network }\end{array}$ & FRA & FDC \\
none & none & none \\
\hline TA & Prune servent-cache & none & none \\
\hline
\end{tabular}

TABLE VII

Describing Freenet Using the ReFEREnCE ARChiteCtURe.

the new node a key space to manage. The Naming servent generates a unique key for files to be stored on freenet using SHA-1 secure hashes. The SHA-1 secure hashes ensure that same key is generated for identical files. Freenet does not allow the resolution of the id to an actual physical address to ensure anonymity of the peer storing the file.

getData(...) and insertData(...) servents share a single servent-cache which is a table of (dataKey, (hostAddress, data)) tuples. The servent cache is pruned by TopologyManager to ensure that its size does not exceed a specified limit. A least recently used algorithm is used to remove the data for a key. The host address is still cached so that the data can be retrieved at a later time if required.

The getData(...) and insertData(...) servent implementations

\begin{tabular}{l|l}
\hline JXTA Protocol & Reference Architecture Elements \\
\hline PDP & ServiceDiscovery, ServiceAdvertisement \\
\hline PRP & Client, Server \\
\hline RVP & Application Servents \\
\hline ERP & Application Servents \\
\hline PIP & Application Servents \\
\hline
\end{tabular}

TABLE VIII

A MAPPING BETWEEN THE JXTA PROTOCOLS AND THE REFERENCE ARCHITECTURE ELEMENTS.

propagate a request to the host (in servent-cache) whose key is closest to the key in the request. The getData(...) request is not propagated further and a response is sent back if the key in the request is already present in the servent-cache along with the data, otherwise the request is forwarded to the host in the servent-cache whose key is closest to the key in the request. The getData(...) response is sent back to the peer which forwarded the request which in turn sends the response back to the peer from which it received the request. The response is not directly sent back to the peer from which the request originates in order to preserve anonymity of the peer interested in the resource. The insertData(...) request is not propagated further and data is added to the servent-cache if the id of the peer is closest to the key in the request than any other key already present in the servent-cache, otherwise the request is forwarded to the host in the servent-cache whose key is closest to the key in the request.

\section{JXTA}

JXTA [38], [18] was conceived by Sun Microsystems Inc and has been developed as an open source project. JXTA is a specification for developing P2P applications. The specifications can be implemented as a framework which can be used for P2P application development. The Project JXTA reference implementation [39] is one such framework. JXTA provides components which can be used by a P2P application. For example, JXTA provides an abstraction called pipes that is similar to BSD socket interface and can be used for communication between peers. JXTA defines the structure of elements like PeerInformation (peer advertisement) and ServiceDetail (service advertisement) shown in Table III. However JXTA does not provides the software architecture of a P2P system.

JXTA specifications define a set of six protocols for P2P applications. JXTA protocols work together to perform services required by a peer. The protocols use XML schema to describe the format of the messages exchanged between peers to perform a service. JXTA is policy agnostic and does not specify how the service provided by a protocol will be implemented. Table VIII shows a mapping between the JXTA protocols and the reference architecture elements. Peer Resolver Protocol (PRP) defines the structure of XML request and response messages which are exchanged between peers. The rest of the protocol messages are embedded within a PRP message. The client and server component in the common runtime can use this as the format of the messages exchanged between 
peers. A peer can advertise and discover resources and services on the overlay network using Peer Discovery Protocol (PDP). The ServiceDiscovery and ServiceAdvertisement elements can exchange messages using the format specified in PDP protocol.

There is no direct mapping possible between the next three protocols and the reference architecture elements. These JXTA protocols can be used by application servents in the reference architecture. The Peer Information protocol (PIP) in JXTA, can be used to inquire the status of a peer. In the reference architecture an application servent which provides the status of a peer can use this protocol. In JXTA the client may use Rendezvous Protocol (RVP) to find peers if the message has to be propagated over the overlay network. The reference architecture does not provides a dedicated servent to find peers that can propagate a message on the overlay network. This has been done to keep the list of core servents minimal. In the reference architecture a servent can use the serventcache or the ServiceDiscovery servent to find peers which can propagate a message over the overlay network. If a direct connection to a destination peer cannot be found then Endpoint Routing Protocol (ERP) is used to find intermediate hosts which can route the information to the destination peer. In the reference architecture routing is the responsibility of the servent handling the message. The reference architecture does not stipulate a specific service that is responsible for finding peers which can be used to route messages and suggests using the ServiceDiscovery component to find peers which can route the message. If the application requires a specific service that can be used to find peers which can be used to propagate messages on the network or find peers which can route a message, then the service has to be implemented as an application servent.

\section{Jabber}

Jabber [29], [4], [30] provides specifications for developing instant messaging (IM) applications. Jabber implementations provide a realization of these specifications. Jabber is intended for IM applications, but the infrastructure provided by it can be used for developing other types of P2P applications.

Table IX shows the application servents that Jabber provides and describes how Jabber implements the core servents. Jabber uses a hybrid P2P architecture. The Jabber server is used for authenticating peers and resolving id to a physical network address. However, peers can exchange data directly by establishing a connection which is brokered using Jabber servers. A new peer connects to the central server to join the overlay network. The peers inform the central server when they leave the overlay network. The central server provides the connecting peer with information (e.g., availability of peer and peer address) about other peers in which the connecting peer is interested. The Jabber server provides three presence related services: subscribeToPresenceInformation(...) which can be used to tell the Jabber server about interest in a peer's (with id peerId) availability information, getSubscribedPeers() gives a list of all the peers in which the peer invoking this service is interested and getPresenceInformation(...) can be used to

\begin{tabular}{|c|c|}
\hline \multicolumn{2}{|c|}{$\begin{array}{l}\text { void receiveMessage(String mesg) } \\
\text { boolean subscribeToPresenceInformation(String peerId) } \\
\text { String[] getSubscribedPeers() } \\
\text { boolean getPresenceInformation(String peerId) }\end{array}$} \\
\hline \multicolumn{2}{|c|}{ Core Servents } \\
\hline Name & Policy \\
\hline OND & central server \\
\hline MM(Join) & online peers \\
\hline MM(Leave) & central server \\
\hline SA & none \\
\hline SD & none \\
\hline $\mathrm{N}(\mathrm{ID})$ & central server \\
\hline N(ID) & central server \\
\hline TA & none \\
\hline
\end{tabular}

THE APPLICATION SERVENTS PROVIDED BY JABBER AND THE POLICIES USED BY JABBER TO IMPLEMENT THE DIFFERENT CORE SERVENTS. THE SERVENTS IN JABBER DO NOT USE A SERVENT CACHE. THE SERVENT IMPLEMENTATIONS IN JABBER DO NOT HAVE ROUTING CAPABILITY.

receive presence information about a peer (with id peerId) on the network. All the peers implement a service called receiveMessage(...) which can be used to send a chat message to them.

\section{Future WORK AND CONCLUSION}

The paper presents a taxonomy of core concerns that a P2P application needs to address. Despite the popularity of $\mathrm{P} 2 \mathrm{P}$ applications there is no reference architecture for the P2P domain. The paper presented a reference architecture for the P2P domain. The reference architecture uses a service-oriented approach that facilitates the development of a software implementation (e.g. a P2P file-sharing application) using the reference architecture because the software implementation can use the support for service-oriented architecture provided by the existing middlewares. The paper validates the reference architecture by describing four prominent $\mathrm{P} 2 \mathrm{P}$ applications and middlewares using the reference architecture. The authors are currently working on implementing a customizable middleware based on the reference architecture that allows control over the core servents policies. Future work could involve developing a catalogue of policies for the core servents.

\section{ACKNOWLEDGMENT}

The work described in this article is funded by the Irish Research Council for Science, Engineering and Technology (IRCSET) under the Basic Research Grants Scheme.

\section{REFERENCES}

[1] S. Saroiu, K. Gummadi, R. Dunn, S. Gribble, and H. Levy, "An analysis of internet content delivery systems," in Proc. of the Fifth Symposium on Operating Systems Design and Implementation (OSDI), 2002.

[2] [Online]. Available: http://www.bittorrent.com

[3] "Kaza," http://www.kazaa.com/us/index.htm.

[4] D. Adams, Programming Jabber, C. Toporek, Ed. O'Reilly and Associates, 2002.

[5] J. Edwards, Peer-to-Peer Programming On Groove. Addison-Wesley, 2002. 
[6] P. C. Len Bass and R. Kazman, Software Architecture In Practise. Addison Wesley, 2000.

[7] R. N. Christine Hofmeister and D. Soni, Applied Software Architecture. Addison-Wesley, 2000.

[8] B. P. Gallagher, "Using the architecture tradeoff analysis method to evaluate a reference architecture: A case study," CMU/SEI, Tech. Rep., 2000.

[9] R. Kazman, L. J. Bass, M. Webb, and G. D. Abowd, "SAAM: A method for analyzing the properties of software architectures," in International Conference on Software Engineering, 1994.

[10] A. E. Hassan and R. C. Holt, "A reference architecture for web servers," in Seventh Working Conference on Reverse Engineering (WCRE'O0), 2000.

[11] "Napster," http://www.napster.com, 1999 version, 1999.

[12] [Online]. Available: http://en.wikipedia.org/wiki/Peer-to-peer

[13] M. Henning and S. Vinoski, Advanced CORBA Programming with $\mathrm{C}++$. Addison Wesley, 1999.

[14] [Online]. Available: http://www.w3.org/TR/soap/

[15] P. A. Bernstein, "Middleware: A model for distributed system services," Communications of the ACM, no. 39(2), pp. 86-98, 1996.

[16] A. Singhai, "Quarterware: A middleware toolkit of software risc components," Ph.D. dissertation, Dept. of Computer Science, University of Illinois, 1999.

[17] W. Grosso, JAVA RMI. O'Reilly, 2001.

[18] B. T. Scott Oaks and L. Gong, JXTA In a Nutshell. O'Reilly and Associates Inc., 2002.

[19] Microsoft, "Introduction to windows peer-to-peer networking," Microsoft Corporation, Tech. Rep., 2003.

[20] H. He, "What is service-oriented architecture?" xml.oreilly.com.

[21] M. S. Pallos, "Service-oriented architecture: A primer," eAI Journal, 2001.

[22] M. P. Papazoglou and D. Georgakopoulos, "Service-oriented computing," Communications of the ACM, October 2003.

[23] J. R. Grady Booch and I. Jacobson, The Unified Modeling Language User Guide. Addison-Wesley Pub Co, 1998.

[24] D. Soni, R. L. Nord, and C. Hofmeister, "Software architecture in industrial applications," in Proceedings of the 17th international conference on Software engineering, 1995.

[25] C. Hofmeister, R. Nord, and D. Soni, "Describing software architecture with uml." in Proceedings of the First Working IFIP Conference on Software Architecture, 1999.

[26] J. Ivers, P. Clements, D. Garlan, R. Nord, B. Schmerl, and J. R. O. Silva, "Documenting component and connector views with uml 2.0," Carnegie Mellon Software Engineering Institute, Tech. Rep., 2004.

[27] D. S. e. a. Milojicic, "Peer-to-peer computing," HP Labs, Tech. Rep., 2002.

[28] A. Singh and M. Haahr, "A survey of p2p middlewares," submitted to EURO-PAR 2005.

[29] "What is jabber?" http://www.jabber.org/about/overview.php.

[30] C. Dodson, "Jabber technical white paper," http://xml.coverpages.org/, Aug 2000.

[31] R. Laddad, AspectJ In Action. Manning Publications Co., 2003.

[32] [Online]. Available: http://www.eclipse.org/aspectj/doc/progguide/index.html

[33] "Making p2p interoperable: The jxta story," http://www106.ibm.com/developerworks.

[34] [Online]. Available: http://www.gnutella.com/

[35] M. Ripeanu, "Peer-to-peer architecture case study: Gnutella network," in First International Conference on Peer-to-Peer Computing (P2P2001), 2001.

[36] I. Clarke, S. G. Miller, T. W. Hong, O. Sandberg, and B. Wiley, "Protecting free expression online with freenet," IEEE Internet Computing, 2002.

[37] [Online]. Available: http://freenetproject.org/

[38] "Jxta v2.0 protocols specification," http://spec.jxta.org/nonav/v1.0/docbook/JXTAProtocols.html.

[39] "Project jxta 20 superpeer virtual network," http://www.jxta.org/project/www/docs/JXTA2.0protocols1.pdf. 